\section{MS7-P8 A Closer Look at the Parallel RNA Double Helix Poly(rA)}

Anna V. Luebben ${ }^{1}$, Jingwei Xie ${ }^{2}$, Nozhat Safaee ${ }^{2}$, Kalle Gehring ${ }^{2}$, George M. Sheldrick ${ }^{1}$, Birger Dittrich ${ }^{3}$

1. Georg-August-University Göttingen Germany

2. McGill University, Montreal Canada

3. Heinrich-Heine University, Düsseldorf Germany

email: aluebbe@gwdg.de

As early as 1961, Rich, Davies, Crick and Watson proposed a parallel double helix RNA structure for poly(rA) on the basis of diffuse fibre diffraction photographs [1], using similar arguments to those used to deduce the antiparallel double helix structure for DNA. It took over 50 years before this could be confirmed by a crystal structure determination [2]. However the solution of this structure by $a b$ initio direct methods required one week on an eight-CPU workstation.

We recently obtained synchrotron data from better quality crystals of significantly higher resolution and completeness. With the new data the structure can be solved in a much shorter time and the data quality appears to be sufficient for a charge density analysis using the Invariom approach [3].

[1] Rich, Davies, Crick and Watson, J. Mol. Biol. 1961, 3, $71-86$.

[2] Safaee, Noronha, Rodionov, Kozlov, Wilds, Sheldrick and Gehring, Angew. Chem. Int. Ed. 2013, 52, $10370-10373$

[3] Dittrich, Hübschle, Pröpper, Dietrich, Stolper and Holstein, Acta Cryst. 2013, B69, 91-104.

Keywords: parallel double helix, RNA, charge density, direct methods
MS7-P9 Structural insights into ObcA, an enzyme for oxalogenesis

Sangkee Rhee ${ }^{1}$, Juntaek $\mathrm{Oh}^{1}$, Ingyu Hwang ${ }^{1}$

1. Seoul National University, KOREA

email: srheesnu@snu.ac.kr

Bacterial quorum sensing (QS), a cell-to-cell communication process in many Proteobacteria, controls the gene expression for bacterial population-wide characteristics, including bioluminescence, motility, and virulence-related factors. Recently, QS has been recognized to provide further benefits at the population level by regulating the production of public goods, the function of which could be beneficial to all members of the group. In Burkholderia species, oxalic acid was recently identified as an excreted public good for the QS-dependent growth. In these species, QS-mediated oxalogenesis via the oxalate biosynthetic component (obc) is a cellular event indispensable for the survival of bacteria in the stationary phase. In $B$. glumae, obc consists of two genes encoding ObcA and $\mathrm{ObcB}$ for coordinating the production of oxalic acid, as well as acetoacetate and $\mathrm{CoA}$, by using oxaloacetate and acetyl-CoA as substrates. In ObcA, oxaloacetate serves as a nucleophile by forming an enolate intermediate mediated by Tyr322 as a general base, which then attacks the thioester carbonyl carbon of acetyl-CoA to yield a tetrahedral adduct between the two substrates. Therefore, ObcA catalyzes its reaction by combining the enolase and acetyltransferase superfamilies, but the presence of the metal-coordination shell and absence of general acid(s) likely produces an unusual tetrahedral CoA adduct as a stable product. These results provide the structural basis for understanding the first step in oxalogenesis and the mechanistic features of ObcA. This work was supported by Next Generation BioGreen 21 program of Rural Development Administration (Plant Molecular Breeding Center) of Republic of KOREA.

Keywords: Enzyme catalysis, oxalate production 